\title{
Stage IIIA Small Intestinal Gastrointestinal Stromal Tumor
}

National Cancer Institute

\section{Source}

National Cancer Institute. Stage IIIA Small Intestinal Gastrointestinal Stromal T umor. NCI

Thesaurus. Code C87833.

Stage IIIA includes: (T1, NO, MO, Mitotic rate: High); (T4, NO, MO, Mitotic rate: Low). T1:

Tumor $2 \mathrm{~cm}$ or less in greatest dimension. T4: Tumor more than $10 \mathrm{~cm}$ in greatest

dimension. N0: No regional lymph node metastasis. M0: No distant metastasis. (AJCC 7th ed.) 